\title{
Quasi-theoretical investigation of four circularly polarized metaloop antennas
}

\author{
Hisamatsu Nakano, Tomoki Abe, and Junji Yamauchi \\ Science and Engineering, Hosei University, Koganei, Tokyo, Japan
}

Received: 28 August 2018 / Accepted: 2 November 2018

\begin{abstract}
Four metamaterial line loop (metaloop) antennas, RND-MTLP-C, RND-MTLP-N, SQR-MTLP-C, and SQR-MTLP-N are investigated to clarify the antenna characteristics. Each metaloop is made up of either a C-type metamaterial line or an N-type metamaterial line, where the dispersion characteristics of the unit cell for the $\mathrm{C}$ - and N-type metamaterial lines are designed to be as similar as possible. The RND-MTLP-C and SQR-MTLP-C act as a dual-band counter circularly polarized antenna across a fast wave frequency region. It is found that, depending on the deviation factor, the SQR-MTLP-N behaves as a tri-band circularly polarized antenna, but the RND-MTLP-N does not have the tri-band characteristic. The radiation pattern, gain, and input characteristic in terms of the VSWR for the four antennas are clarified.
\end{abstract}

Keywords: Metaloop antennas / circularly polarized wave / polarization / dual-band operation / tri-band operation

\section{Introduction}

A conventional loop antenna, shown in Figure 1a, generates a linearly polarized (LP) beam in the two directions normal to the antenna plane (i.e. $\pm z$ directions) at a frequency that leads to a loop circumference of one wavelength [1]. This bidirectional beam can be changed to a unidirectional beam using a conducting plane (reflector), where the spacing between the loop and the reflector is chosen to be one-quarter wavelength, so that the direct radiation from the loop toward the positive $z$-direction and the radiation reflected by the reflector constructively add in the positive $z$-direction.

The above-mentioned unidirectional LP beam can be changed to a circularly polarized (CP) beam by adding perturbation elements to the loop, as shown in Figure 1b [2]. The rotational sense of the $\mathrm{CP}$ radiation in this situation is uniquely determined by the location of the feed point and the perturbation elements. In other words, a conventional loop antenna with a fixed feed point and perturbation elements only radiates either a left-handed or a right-handed $\mathrm{CP}$ beam.

However, with increasing investigation of metamaterial-based antennas [3-6], recent research has revealed that a single antenna with a fixed feed point can radiate a left-handed $\mathrm{CP}$ beam in a specific frequency band and a

\footnotetext{
*orresponding author: hymat@hosei.ac.jp
}

right-handed $\mathrm{CP}$ wave in a different frequency band (dualband counter $\mathrm{CP}$ radiation). This fixed feed point antenna is made by spirally winding a metamaterial line (metaline) that is realized using the concept of a composite right- and left-handed transmission line [7-9], as shown in Figure 1c; the antenna is called the metaspiral antenna [10]. The metaspiral antenna consists of an arm composed of numerous turns printed on a thin dielectric substrate backed by a ground plane. The antenna height above the ground plane is very small - on the order of $1 / 100$ wavelength.

A question arises as to whether dual-band counter $\mathrm{CP}$ radiation can be realized when the number of turns for the spirally wound arm is reduced to a single turn and subsequently the single turn arm is formed into a loop structure. The answer to this question has appeared in reference [11], where the dual-band counter $\mathrm{CP}$ radiation from a square metamaterial line loop (metaloop) antenna is described.

A counterpart to the square metaloop exists and is called the round metaloop. A further question arises as to whether the behaviour of the $\mathrm{CP}$ radiation for the round metaloop (RND-MTLP) is the same as that for the square metaloop (SQR-MTLP). As will be found later, the behaviour of the $\mathrm{CP}$ radiation depends on the type of metaline that constitutes the RND- and SQR-MTLP antennas.

This paper presents a theoretical investigation of the behaviour of the $\mathrm{CP}$ radiation using two types of metaline: a C-type metaline and an N-type metaline. The 


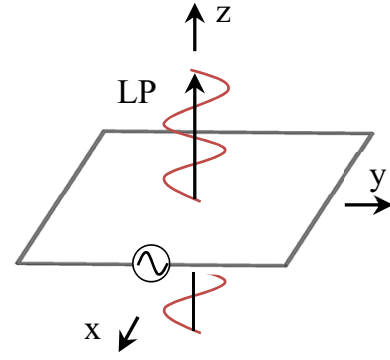

(a)

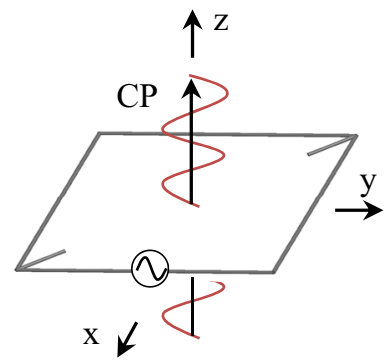

(b)

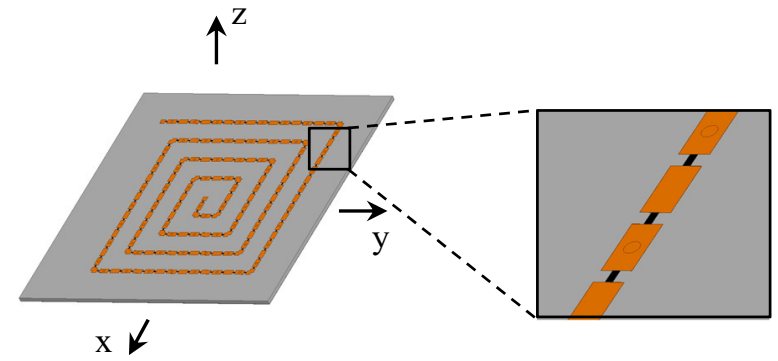

(c)

Fig. 1. (a) Conventional loop antenna that forms linearly polarized bidirectional radiation. (b) Loop antenna with perturbation elements that form circularly polarized radiation. (c) Metaspiral antenna that forms circularly polarized radiation.

investigation is performed for four metaloops: RNDMTLP-C, RND-MTLP-N, SQR-MTLP-C and SQRMTLP-N, where the last letters $(\mathrm{C}$ and $\mathrm{N})$ denote the type of metaline that is used for the metaloop, the $\mathrm{C}$-type or the N-type.

Five sections constitute this paper. Section 2 presents $\mathrm{C}$ - and N-type metalines and their radiation characteristics. Section 3 discusses the RND-MTLP-C and RNDMTLP-N antennas, which approximately realize the radiation models round model- $C$ and round model- $N$, respectively. Similarly, Section 4 discusses the SQRMTLP-C and SQR-MTLP-N antennas, which approximately realize the radiation models square model- $C$ and square model- $N$, respectively. Section 5 summarizes the obtained results.

\section{Metamaterial lines (metalines)}

Figure 2a shows a metamaterial line, referred to as a C-type metaline, based on the concept of a conventional composite right- and left-handed (CRLH) transmission line [7-9], while Figure $2 \mathrm{~b}$ shows a novel metaline, referred to as an N-type metaline. Both are printed on a dielectric substrate of relative permittivity $\varepsilon_{\mathrm{r}}$ and thickness $B$. The substrate is backed by a conducting ground plane (GP). Point $F$ is the feed point. The metaline end point, $T$, is terminated through resistive load $R_{\mathrm{B}}$.

The C-type metaline is composed of periodically arrayed subwavelength segments of width $w$ and length $p_{0}$ with
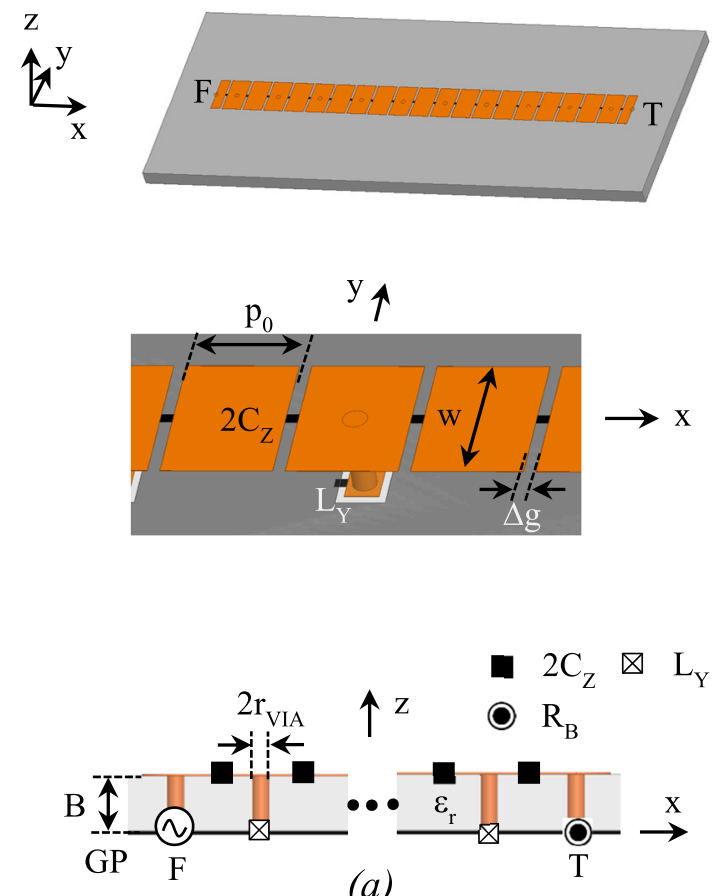

(a)
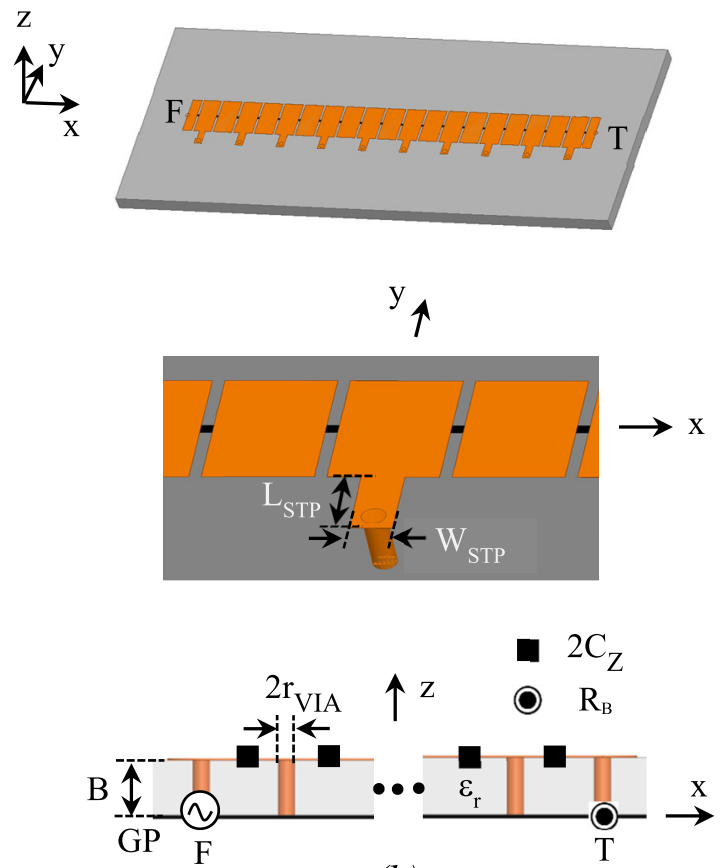

(b)

Fig. 2. Metalines. (a) Perspective view (upper), exploded view (middle) and side view (bottom) of a C-type metaline. (b) Perspective view (upper), exploded view (middle) and side view (bottom) of an N-type metaline.

separation $\Delta g$. The region across length $2\left(p_{0}+\Delta g\right) \equiv p$ is called the unit cell. The central segment of the unit cell is shorted to the ground plane through inductance $L_{Y}$ using a conducting vertical probe (via) of radius $r_{\text {via }}$. Neighbouring subwavelength segments are connected 


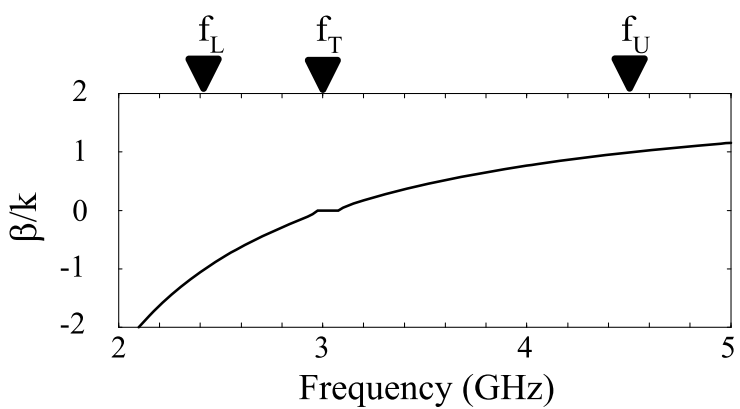

(a)

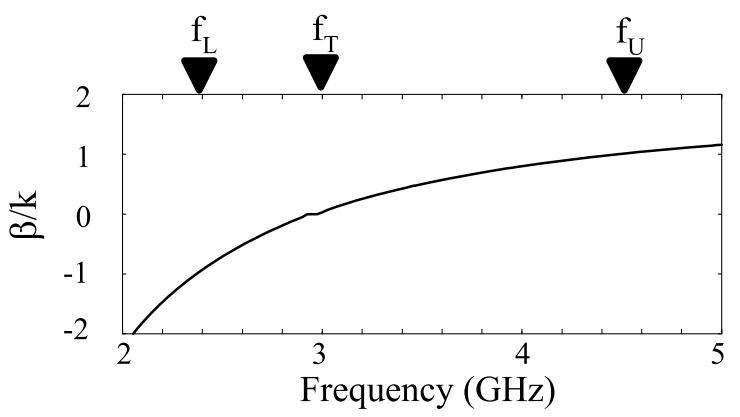

(b)

Fig. 3. Dispersion diagram for the unit cell. (a) C-type metaline. (b) N-type metaline.

Table 1. Parameters.

\begin{tabular}{llll}
\hline Symbol & Value & Symbol & Value \\
\hline$\varepsilon_{\mathrm{r}}$ & 2.6 & $r_{\text {VIA }}$ & $0.5 \mathrm{~mm}$ \\
$B$ & $3.2 \mathrm{~mm}$ & $W_{\text {STP }}$ & $1.0 \mathrm{~mm}$ \\
$w$ & $6.6 \mathrm{~mm}$ & $L_{\mathrm{STP}}$ & $4.5 \mathrm{~mm}$ \\
$p_{0}$ & $4.5 \mathrm{~mm}$ & $2 C_{\mathrm{Z}}$ & $1.2 \mathrm{pF}$ \\
$\Delta g$ & $0.5 \mathrm{~mm}$ & $L_{\mathrm{Y}}$ & $1.8 \mathrm{nH}$ \\
\hline
\end{tabular}

through a capacitive element, $2 C_{\mathrm{Z}}$, which is inserted into the gap between segments.

The N-type metaline in Figure $2 \mathrm{~b}$ differs from the C-type metaline in that the unit cell's central segment does not have a conducting vertical probe; instead, it has a horizontal strip of width $W_{\mathrm{STP}}$ and length $L_{\mathrm{STP}}$ that extends from the central subwavelength segment in the negative $y$-direction, with the end of the strip being shorted to the ground plane. As seen later, the N-type metaline acts as a circularly polarized radiation element, as opposed to the C-type metaline, which acts as a linearly polarized radiation element.

Figure 3 shows the dispersion diagram of the unit cell for C-type and N-type metalines of infinite length. The parameters used are summarized in Table 1. These two metalines are designed to have dispersion characteristics that are as similar as possible. The notation is as follows: $k(=2 \pi / \lambda$ with free space wavelength $\lambda)$ and $\beta\left(=2 \pi / \lambda_{\mathrm{g}}\right.$ with guided wavelength $\left.\lambda_{\mathrm{g}}\right)$ are the propagation phase

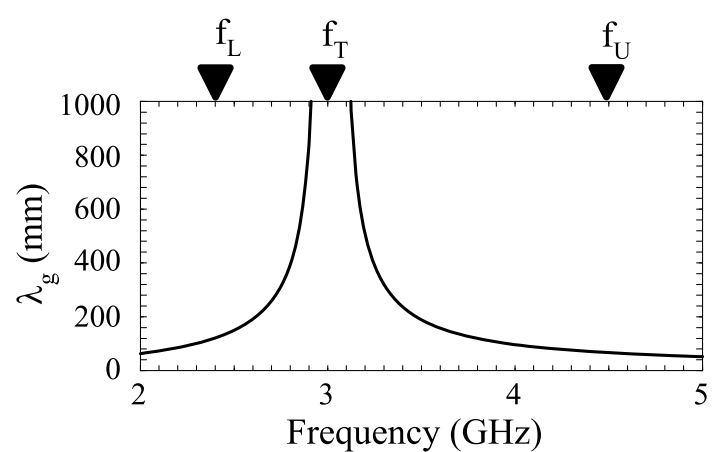

(a)

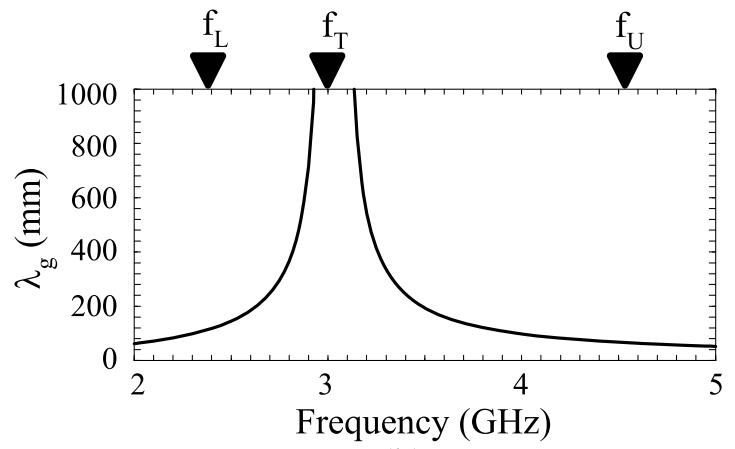

(b)

Fig. 4. Guided wavelength $\lambda_{\mathrm{g}}$ for the unit cell as a function of frequency. (a) C-type metaline. (b) N-type metaline.

constants in free space and along the metaline, respectively; $f_{\mathrm{T}}$ is the transition frequency; and $f_{\mathrm{L}}$ and $f_{\mathrm{U}}$ are the lower and upper band edge frequencies for a fast wave, respectively. Note that the dispersion in terms of guided wavelength, $\lambda_{\mathrm{g}}$, is shown in Figure 4.

The radiation from $\mathrm{C}$-type and $\mathrm{N}$-type metalines of 10 cells $\left(N_{\text {cell }}=10\right)$ in length is shown in Figure 5 , where the parameters in Table 1 are used; the end of the metaline is terminated with a resistive load of $R_{\mathrm{B}}=60$ ohms. Propagation phase constant $\beta$ at frequencies below transition frequency $f_{\mathrm{T}}(=3 \mathrm{GHz})$ is negative and hence both metalines form radiation beams in the backward direction, while $\beta$ at frequencies above $f_{\mathrm{T}}$ is positive and hence both metalines form radiation beams in the forward direction.

The current along the periodically arrayed subwavelength segments for the C-type metaline flows in the longitudinal direction, i.e. the $x$-direction, and the radiation is linearly polarized (LP), as shown in Figure $5 \mathrm{a}$, where the radiation field is decomposed into the $\theta$-directed electric field component, $E_{\theta}$, and the $\phi$-directed electric field component, $E_{\phi}$. This result is expected from conventional CRLH transmission line theory [7-9].

In contrast, the radiation from the N-type metaline is circularly polarized (CP), as shown in Figure 5b, where the radiation field is decomposed into the left-handed CP electric field component (LHCP wave), $E_{\mathrm{L}}$, and the righthanded $\mathrm{CP}$ electric field component (RHCP wave), $E_{\mathrm{R}}$. Note that $E_{\mathrm{L}}$ is dominant, i.e. $E_{\mathrm{L}}$ is the co-polarized component and $E_{\mathrm{R}}$ is the cross-polarized component. This 


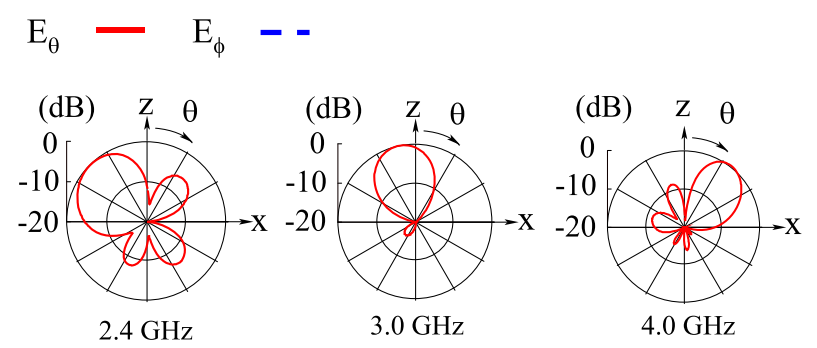

(a)

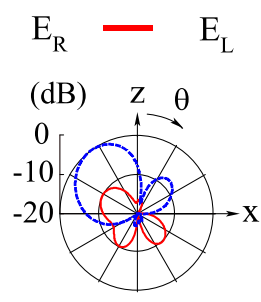

$2.4 \mathrm{GHz}$



(b)
Fig. 5. Radiation from a 10 -cell metaline $\left(N_{\text {cell }}=10\right)$. The parameters in Table 1 are used, with $R_{\mathrm{B}}=60 \Omega$. (a) C-type metaline. (b) N-type metaline.

is attributed to the fact that the unit cell for the N-type metaline has currents in the $x$-direction (longitudinal direction) and negative $y$-direction (the strip direction), where the negative $y$-directed current is delayed by $90^{\circ}$ with respect to the $x$-directed current, resulting in LHCP radiation.

\section{Round metaloop antenna}

\subsection{Round model-C}

The principle for forming $\mathrm{CP}$ radiation in the broadside direction (positive $z$-direction) is to be considered here. Figure 6 shows an antenna model, called round model- $C$, where the loop is made of a curved narrow width line and has radius $r_{\mathrm{LP}}$.

We express the current at distance $s^{\prime}$, measured from point $F$ in the counter-clockwise direction, as $\boldsymbol{I}\left(s^{\prime}\right)=$ $I_{0} e^{-j \beta s^{\prime}} \hat{s}$, where $I_{0}$ is the amplitude; $\hat{s}$ is the unit vector tangential to the loop. Note that the time dependence, $e^{j \omega t}$, is omitted.

For deriving the radiation field for the loop, the following assumptions are made: (1) current $\boldsymbol{I}\left(s^{\prime}\right)$ of phase constant $\beta\left(\beta=2 \pi / \lambda_{\mathrm{g}}>0\right.$ or $\left.\beta=-2 \pi / \lambda_{\mathrm{g}}<0\right)$ is a travelling current without attenuation, i.e. $I_{0}$ is constant; (2) there is no reflection current flowing towards feed point $F$, i.e. unidirectional current; (3) there is no mutual coupling between current elements at different positions.

Using unit vectors $\hat{x}$ and $\hat{y}$ with rectangular coordinates, unit vector $\hat{s}$ is transformed into

$$
\hat{s}=-\sin \Phi\left(s^{\prime}\right) \hat{x}+\cos \Phi\left(s^{\prime}\right) \hat{y}
$$

where $\Phi\left(s^{\prime}\right) \equiv s^{\prime} / r_{\text {LP }}$ is the azimuth angle for current $\boldsymbol{I}\left(s^{\prime}\right)$.

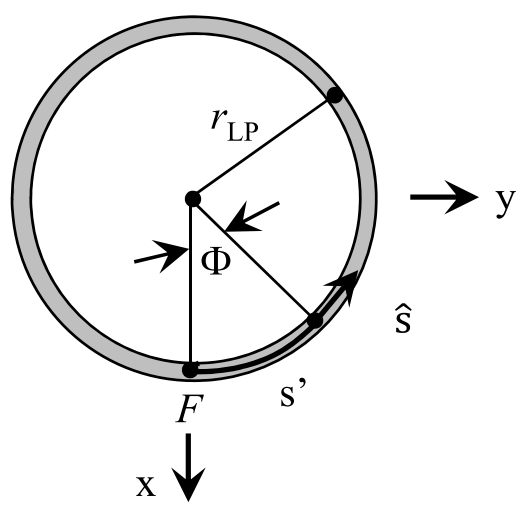

Fig. 6. Round model-C.

The electric field in the spherical coordinate system, $\mathbf{E}$ $(R, \theta, \phi)$, is calculated using the following equation [12]:

$$
\mathbf{E}=-\frac{j \omega \mu}{4 \pi} \frac{e^{-j k R}}{R} \int_{0}^{L} \boldsymbol{I}\left(s^{\prime}\right) e^{j k \hat{R} \cdot \boldsymbol{r}^{\prime}\left(s^{\prime}\right)} d s^{\prime}
$$

where $\omega(=2 \pi f)$ is the angular frequency at frequency $f$; $\mu$ is the permeability in free apace; $k(=2 \pi / \lambda)$ is the propagation phase constant in free space, as already defined; $L=2 \pi r_{\mathrm{LP}} \equiv n \lambda_{\mathrm{g}}$ is the loop circumference, where $n$ is a positive real number; $\hat{R}$ is the unit vector in the $R$-direction of the spherical coordinate system; $\boldsymbol{r}^{\prime}\left(s^{\prime}\right)$ is the position vector from the coordinate origin to a current source point of $s^{\prime} ;\left|\boldsymbol{r}^{\prime}\left(s^{\prime}\right)\right|=r_{\mathrm{LP}}$.

Substituting the assumed current, $\boldsymbol{I}\left(s^{\prime}\right)$, into equation (2), we obtain the field in the broadside direction, $\left.\mathbf{E}_{\mathrm{Brd}}\right|^{n}(R, \theta=0, \phi)$, as

$$
\left.\mathbf{E}_{\mathrm{Brd}}\right|^{n}=-\left.\frac{j \omega \mu}{4 \pi} \frac{e^{-j k R}}{R} \mathbf{A}_{\mathrm{s}}\right|^{n}
$$

where

$$
\begin{aligned}
\left.\mathbf{A}_{\mathrm{s}}\right|^{n}= & \frac{I_{0}}{2} \cdot \frac{n \lambda_{\mathrm{g}}}{j 2 \pi} \cdot\left[\left.K^{+}\right|^{n} \cdot(j \hat{x}+\hat{y})\right. \\
& \left.+\left.K^{-}\right|^{n} \cdot(-j \hat{x}+\hat{y})\right]
\end{aligned}
$$

with

$$
\begin{aligned}
& \left.K^{+}\right|^{n}=\frac{1}{1 \mp n}\left(e^{j(1 \mp n) 2 \pi}-1\right) \quad \text { for } \beta \gtrless 0 \\
& \left.K^{-}\right|^{n}=\frac{1}{-1 \mp n}\left(e^{j(-1 \mp n) 2 \pi}-1\right) \quad \text { for } \beta \gtrless 0
\end{aligned}
$$

$\left.\mathbf{A}_{\mathrm{S}}\right|^{n}$ in equation (4) is referred to as the $\hat{s}$-current polarization vector, which is composed of an RHCP component $(j \hat{x}+\hat{y})$ and an LHCP component, $(-j \hat{x}+\hat{y})$. It is found that a $\mathrm{CP}$ component (either an RHCP component or an LHCP component) is obtained when $n$ is an integer. If $n$ is not an integer, then $\left.\mathbf{A}_{\mathbf{S}}\right|^{n}$ becomes elliptically polarized. 


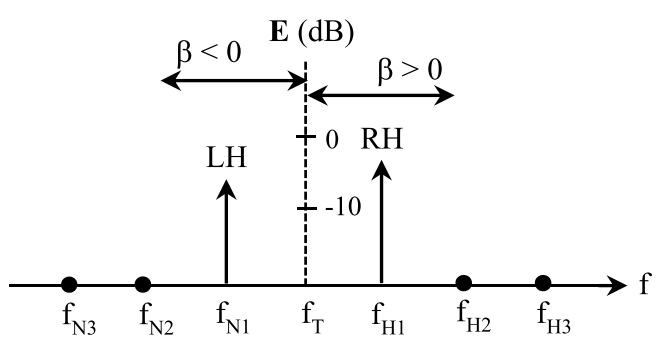

Fig. 7. Broadside radiation $\left.\mathbf{E}_{\mathrm{Brd}}\right|^{\mathrm{n}}=\mathbf{E}$ for round model-C.

Our purpose is to obtain broadside CP radiation, and hence $n$ is chosen to be an integer.

For $n=1$, equations (5) and (6) become

$$
\begin{aligned}
& \left.K^{+}\right|^{n=1}= \begin{cases}j 2 \pi & \text { for } \beta \gtrless 0, \\
0 & \end{cases} \\
& \left.K^{-}\right|^{n=1}= \begin{cases}0 & \text { for } \beta \gtrless 0 . \\
j 2 \pi & \end{cases}
\end{aligned}
$$

Consequently, equation (4) yields

$\left.\mathbf{A}_{\mathrm{S}}\right|^{n=1}= \begin{cases}\frac{I_{0}}{2} \lambda_{\mathrm{g}}(j \hat{x}+\hat{y}) & \text { for } \beta>0 \\ \frac{I_{0}}{2} \lambda_{\mathrm{g}}(-j \hat{x}+\hat{y}) & \text { for } \beta<0 .\end{cases}$

At the frequency defined by $(\beta>0, n=1)$, called the first Hion frequency and denoted as $f_{\mathrm{H} 1}$, the broadside radiation field, $\left.\mathbf{E}_{\mathrm{Brd}}\right|^{n=1}$ in equation (3) is RHCP because of the relationship in equation $(9 \mathrm{a})$. In contrast, at the frequency defined by $(\beta<0, n=1)$, called the first Nion frequency, $f_{\mathrm{N} 1},\left.\mathbf{E}_{\mathrm{Brd}}\right|^{n=1}$ is LHCP because of the relationship in equation $(9 \mathrm{~b})$. The polarization for $n=1$ can also be explained qualitatively, taking the sign of $\beta$ into consideration: when $\beta$ is positive, phase $-\beta s^{\prime}$ along the loop from feed point $F$ is negative and regressive as with a conventional natural loop, i.e. the current flows counterclockwise, resulting in RHCP radiation. On the other hand, when $\beta$ is negative, phase $-\beta s^{\prime}$ is positive and progressive. Hence, the current behaves as if it flows clockwise, resulting in $\mathrm{LHCP}$ radiation.

When $n=2$ and 3 , equations (5) and (6) become

$$
\begin{array}{ll}
\left.K^{+}\right|^{n=2,3}=0, & \text { for } \beta \gtrless 0 \\
\left.K^{-}\right|^{n=2,3}=0, & \text { for } \beta \gtrless 0
\end{array}
$$

and hence equation (4) yields

$$
\left.\mathbf{A}_{\mathrm{S}}\right|^{n=2,3}=0 \quad \text { for } \beta \gtrless 0 .
$$

To summarize, the $\hat{\text { s}}$-current polarization vector, $\left.\mathbf{A}_{\mathrm{s}}\right|^{n}$, is zero at the second Hion frequency, $f_{\mathrm{H} 2}$, defined by $(\beta>0$, $n=2)$ and the second Nion frequency, $f_{\mathrm{N} 2}$, defined by $(\beta<0, n=2)$, resulting in no broadside radiation: $\left.\mathbf{E}_{\text {Brd }}\right|^{n=2,3}=0$. Zero broadside radiation also occurs

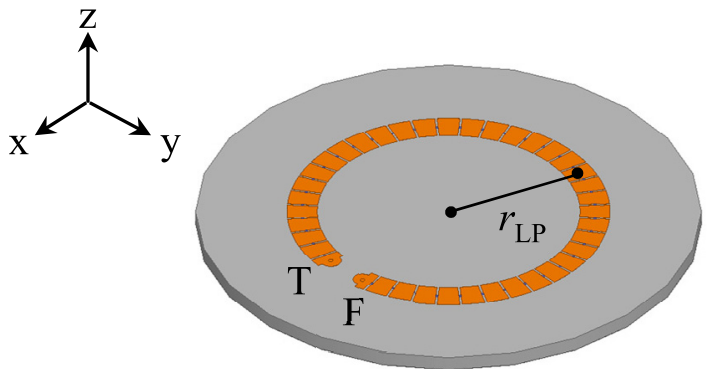

Fig. 8. Round metaloop-C antenna (RND-MTLP-C), made by curving the C-type metaline shown in Figure 2a, where $N_{\text {cell }}=19$.

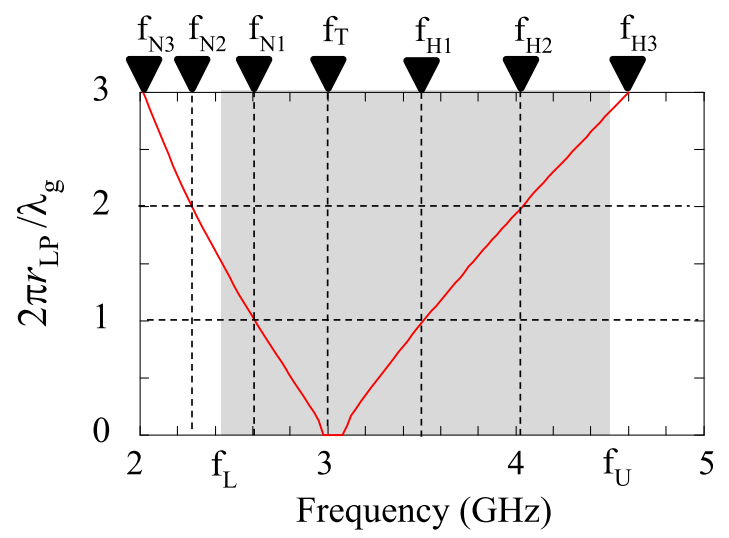

Fig. 9. Normalized loop circumference $2 \pi r_{\mathrm{LP}} / \lambda_{\mathrm{g}}$ as a function of frequency, where the radius of the metaloop is $r_{\mathrm{LP}}=31.8 \mathrm{~mm}$.

at the third Hion frequency, $f_{\mathrm{H} 3}$, and the third Nion frequency defined by $(\beta>0, n=3)$ and $(\beta<0, n=3)$, respectively. The above discussion leads to $\left.\mathbf{E}_{\mathrm{Brd}}\right|^{n}$ shown in Figure 7.

\subsection{Round metaloop-C antenna (RND-MTLP-C): approximation for round model-C}

RHCP radiation and LHCP radiation in the broadside direction are realized here by using a single antenna under the condition that the location of feed point $\mathrm{F}$ is fixed. For this, the principle in Section 3.1 is implemented by curving the $\mathrm{C}$-type metaline in Figure 2a, where the radius is $r_{\mathrm{LP}}$, as shown in Figure 8. The antenna is referred to as the round metaloop-C antenna or RND-MTLP-C. Note that loop end $T$ is terminated through resistive load $R_{\mathrm{B}}(=60 \Omega)$ to the ground plane. The parameter values $\left(w, p_{0}, \Delta \mathrm{g}, \varepsilon_{\mathrm{r}}, B, 2 C_{\mathrm{Z}}, L_{\mathrm{Y}}, r_{\mathrm{via}}\right)$ for this metaloop are the same as those shown in Table 1.

The following simulation/analysis is performed using a full-wave analysis tool $\left(\mathrm{HFSS}^{1}\right)$, whose results include effects of a finite-length non-straight metaline structure on the current: phase constant (and hence the guided wavelength) and attenuation constant along the loop, and reflection from the loop end. These effects, which are not

\footnotetext{
$\overline{{ }^{1} \text { http://www.ansoft.com/products/hf/hfss }}$
} 


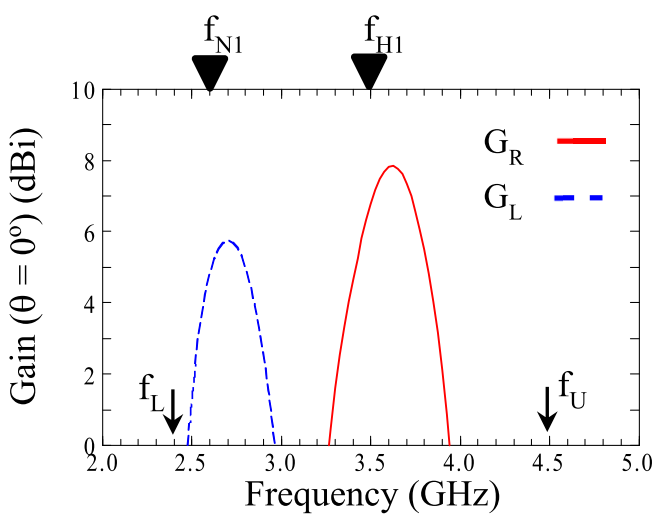

(a)

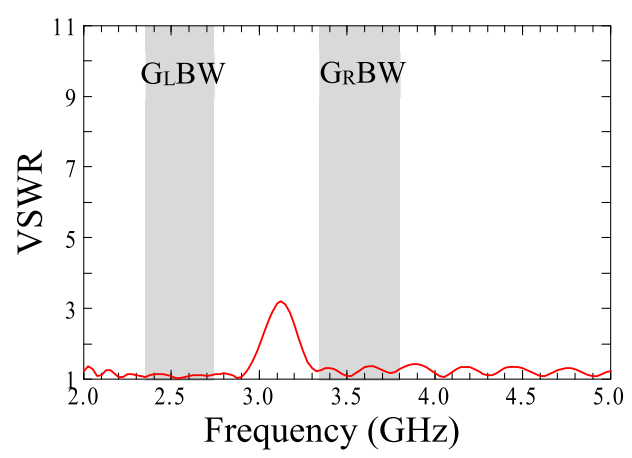

(b)

Fig. 10. Frequency response for the round metaloop-C antenna (RND-MTLP-C). (a) LHCP and RHCP gains. (b) VSWR.

included in the round model-C, change the intensity of the broadside radiation $\mathbf{E}$ presented by the round model-C; however, the discussed behaviour of polarization is still valid. This holds true for RND-MTLP-N in Section 3.4, SQR-MTLP-C in Section 4.2 and SQR-MTLP-N in Section 4.4, against round model-N, square model-C and square model-N, respectively.

The normalized loop circumference, $2 \pi r_{\mathrm{LP}} / \lambda_{\mathrm{g}}$, as a function of frequency is shown in Figure 9, where metaloop radius $r_{\mathrm{LP}}$ is chosen to be $31.8 \mathrm{~mm}$ and $\lambda_{\mathrm{g}}$ in Figure $4 \mathrm{a}$ is used. The shaded frequency region of $f_{\mathrm{L}}$ to $f_{\mathrm{U}}$ shows a fast wave region, where the radiation occurs.

Figure 10a shows the frequency response for the gain, where $G_{\mathrm{L}}$ denotes the gain for an LHCP wave, referred to as the LHCP gain, and $G_{\mathrm{R}}$ denotes the gain for an RHCP wave, referred to as the RHCP gain. As expected from the results in Figure 7, the gain is maximal at frequency $f_{\text {GLmax }}$ near the first Nion frequency, $f_{\mathrm{N} 1}=2.60 \mathrm{GHz}\left(f_{\mathrm{GLmax}}=\right.$ $2.70 \mathrm{GHz} \approx f_{\mathrm{N} 1}$ ), and $f_{\mathrm{GRmax}}$ near the first Hion frequency, $f_{\mathrm{H} 1}=3.50 \mathrm{GHz}\left(f_{\mathrm{GRmax}}=3.60 \mathrm{GHz} \approx f_{\mathrm{H} 1}\right)$. Note that the input characteristic in terms of the VSWR illustrated in Figure $10 \mathrm{~b}$ is desirably small within the $G_{\mathrm{L}}$ band $\left(\mathrm{G}_{\mathrm{L}} \mathrm{BW}\right)$ and $G_{\mathrm{R}}$ band $\left(G_{\mathrm{R}} \mathrm{BW}\right)$, as defined by a 3 -dB gain-drop criterion.

Figure 11 shows the radiation patterns when gains $G_{\mathrm{L}}$ and $G_{\mathrm{R}}$ are maximal, where the radiation field is decomposed into the LHCP wave component, $E_{\mathrm{L}}$, and the RHCP wave component, $E_{\mathrm{R}}$. It is found that the half-power

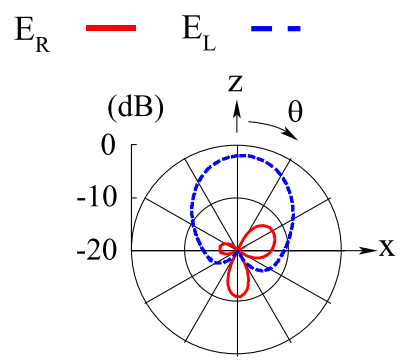

(a)

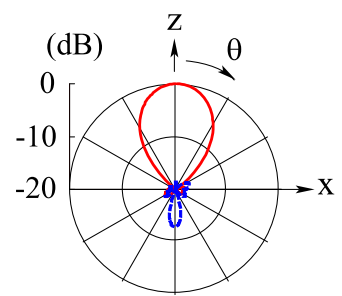

(b)
Fig. 11. Normalized radiation patterns for the round metaloop-C antenna when gains $G_{\mathrm{L}}$ and $G_{\mathrm{R}}$ are maximal. (a) At $f_{\mathrm{GLmax}}=$ $2.70 \mathrm{GHz}$. (b) At $f_{\mathrm{GRmax}}=3.60 \mathrm{GHz}$.

beam-width (HPBW) for $E_{\mathrm{L}}$ is wider than that for $E_{\mathrm{R}}$. This is attributed to the fact that the antenna size relative to the operating free-space wavelength (i.e. the electrical antenna size) at $f_{\text {GLmax }}$ is smaller than that at $f_{\text {GRmax }}$.

\subsection{Round model- $\mathrm{N}$}

The model shown in Figure 12 is referred to as round model- $N$, where the current on the loop made of a curved wide width line is expressed as $\boldsymbol{I}\left(s^{\prime}\right)=I_{0}(\hat{s}-j q \hat{t}) e^{-j \beta s^{\prime}} ; q$ is called the deviation factor and is a function of frequency $f: q=q(f)$. The assumptions for this current are the same as those for round model-C in Section 3.1.

Vector $\hat{t}$ is the unit vector perpendicular to $\hat{s}$ and is given as

$$
\hat{t}=\cos \Phi\left(s^{\prime}\right) \hat{x}+\sin \Phi\left(s^{\prime}\right) \hat{y} .
$$

Note that the $\hat{t}$-directed current has a $90^{\circ}$ phase delay relative to the $\hat{s}$-directed current and travels along the loop with phase constant $\beta$.

Substituting current $\boldsymbol{I}\left(s^{\prime}\right)$ into equation (2), the electric field in the broadside direction, $\left.\mathbf{E}_{\mathrm{Brd}}\right|_{q} ^{n}(R, \theta=0, \phi)$, is formulated as

$$
\left.\mathbf{E}_{\mathrm{Brd}}\right|_{q} ^{n}=-\left.\frac{j \omega \mu}{4 \pi} \frac{e^{-j k R}}{R} \mathbf{A}_{\mathrm{st}}\right|_{q} ^{n}
$$

where polarization vector $\left.\mathbf{A}_{\text {st }}\right|_{q} ^{n}$ generated by $\hat{s}$ - and $\hat{t}$-directed currents is

$$
\begin{aligned}
\left.\mathbf{A}_{\mathrm{st}}\right|_{q} ^{n}= & \frac{I_{0}}{2} \cdot \frac{n \lambda_{\mathrm{g}}}{j 2 \pi} \cdot\left[\left.(1-q) K^{+}\right|^{n} \cdot(j \hat{x}+\hat{y})\right. \\
& \left.+\left.(1+q) K^{-}\right|^{n} \cdot(-j \hat{x}+\hat{y})\right] .
\end{aligned}
$$

Note that $\left.K^{+}\right|^{n}$ and $\left.K^{-}\right|^{n}$ are already defined by equations (5) and (6), respectively.

The current for a deviation factor of $q=0$ is $\boldsymbol{I}\left(s^{\prime}\right)=$ $I_{0} e^{-j \beta s^{\prime}} \hat{s}$ and polarization vector $\left.\mathbf{A}_{\text {st }}\right|_{q=0} ^{n}$ is the same as $\left.\mathbf{A}_{\mathrm{s}}\right|^{n}$, i.e. $\left.\mathbf{A}_{\mathrm{st}}\right|_{q=0} ^{n}=\left.\mathbf{A}_{\mathrm{s}}\right|^{n}$. This allows formation of LHCP radiation at first Nion frequency $f_{\mathrm{N} 1}$ and $\mathrm{RHCP}$ radiation at first Hion frequency $f_{\mathrm{H} 1}$, as with round model-C when $n=1$. 


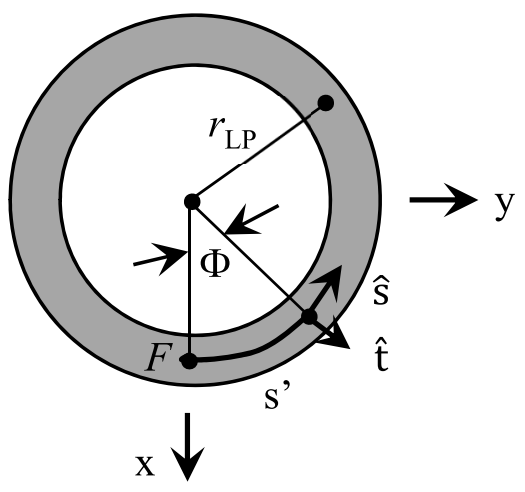

Fig. 12. Round model-N.

$\left.\mathrm{K}^{+}\right|^{n}$ and $\left.\mathrm{K}^{-}\right|^{n}$ when $n=2$ and 3 are zero, as shown by equations (10) and (11), respectively, and hence equation (15) becomes

$$
\left.\mathbf{A}_{\text {st }}\right|_{q=0} ^{n=2,3}=0 \quad \text { for } \beta \gtrless 0,
$$

resulting in no broadside radiation: $\left.\mathbf{E}_{\mathrm{Brd}}\right|_{q=0} ^{n=2,3}=0$, which holds true for integers $n \geq 4$. The broadside radiation, $\left.\mathbf{E}_{\mathrm{Brd}}\right|_{q=0} ^{n}$, is summarized in Figure 13a, which is the same as Figure 7 .

When $q=1$, equation (15) yields

$$
\left.\mathbf{A}_{\mathrm{st}}\right|_{q=1} ^{n}=\left.I_{0} \cdot \frac{n \lambda_{\mathrm{g}}}{j 2 \pi} K^{-}\right|^{n} \cdot(-j \hat{x}+\hat{y}) .
$$

Hence, equation (17) for $n=1$ becomes

$$
\left.\mathbf{A}_{\mathrm{st}}\right|_{q=1} ^{n=1}=\left\{\begin{array}{c}
0 \\
I_{0} \lambda_{\mathrm{g}}(-j \hat{x}+\hat{y})
\end{array} \quad \text { for } \beta \gtrless 0 .\right.
$$

This means that round model-N forms an LHCP wave in the broadside direction at first Nion frequency $f_{\mathrm{N} 1}$ (the frequency satisfies the conditions $\beta<0$ and $n=1$ ); however, round model-N does not form an RHCP wave in the broadside direction at first Hion frequency $f_{\mathrm{H} 1}$ (the frequency satisfies conditions $\beta>0$ and $n=1$ ) as compared to round model-C.

Note that equation (17) for integers $n=2$ and 3 becomes zero,

$$
\left.\mathbf{A}_{\mathrm{st}}\right|_{q=1} ^{n=2,3}=0, \quad \text { for } \beta \gtrless 0,
$$

and hence no broadside radiation is formed, as with round model-C. This also happens at the $n$th Hion frequency, $f_{\mathrm{Hn}}$, and the $n$th Nion frequency, $f_{\mathrm{Nn}}$, for integers $n \geq 4$.

The broadside radiation, $\left.\mathbf{E}_{\mathrm{Brd}}\right|_{q=1} ^{n}$, is illustrated in Figure 13b.

Next, a situation where deviation factor $q$ is neither 0 nor 1 is considered. As one example, a value of $q=0.5$ is arbitrarily chosen. Then, equation (15) becomes

$$
\begin{aligned}
\left.\mathbf{A}_{\mathrm{st}}\right|_{q=0.5} ^{n}= & I_{0} \cdot \frac{n \lambda_{\mathrm{g}}}{j 2 \pi} \cdot\left[\left.\frac{1}{4} K^{+}\right|^{n} \cdot(j \hat{x}+\hat{y})\right. \\
& \left.+\left.\frac{3}{4} K^{-}\right|^{n} \cdot(-j \hat{x}+\hat{y})\right] .
\end{aligned}
$$

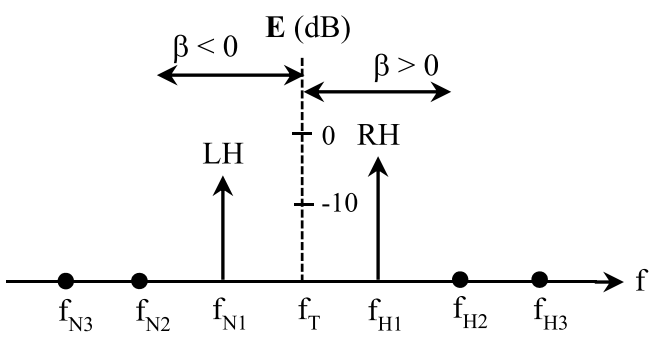

(a)

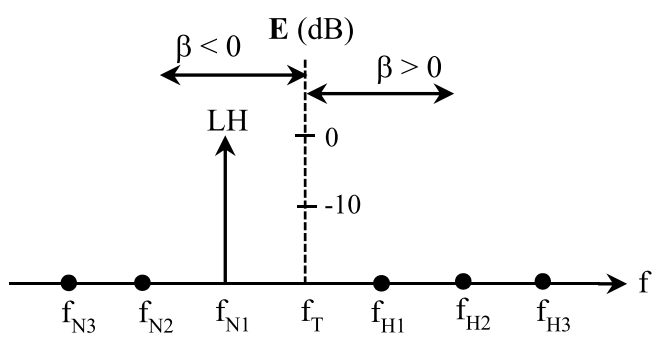

(b)

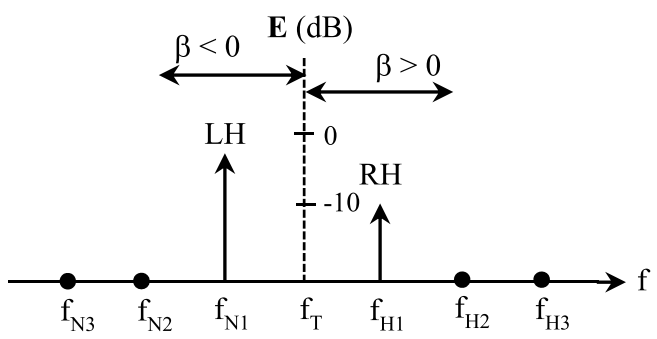

(c)

Fig. 13. $\left.\mathbf{E}_{\mathrm{Brd}}\right|_{q} ^{n}=\mathbf{E}$ for round model-N. (a) $q=0$. (b) $q=1$. (c) $q=0.5$.

At first Nion frequency $f_{\mathrm{N} 1}$ (where $\left.\beta<0, n=1\right),\left.K^{+}\right|^{n=1}=0$ from equation (7) and $\left.K^{-}\right|^{n=1}=j 2 \pi$ from equation (8), and hence equation (20) yields

$$
\left.\mathbf{A}_{\mathrm{st}}\right|_{q=0.5} ^{n=1}=\frac{3}{4} I_{0} \lambda_{\mathrm{g}} \cdot(-j \hat{x}+\hat{y}), \quad \text { for } \beta<0,
$$

leading to LHCP broadside radiation for $\left.\mathbf{E}_{\text {Brd }}\right|_{q=0.5} ^{n=1}$. At first Hion frequency $f_{\mathrm{H} 1}$ (where $\left.\beta>0, n=1\right),\left.K^{+}\right|^{n=1}=j 2 \pi$ from equation (7) and $\left.K^{-}\right|^{n=1}=0$ from equation (8), and hence equation (20) yields

$$
\left.\mathbf{A}_{\mathrm{st}}\right|_{q=0.5} ^{n=1}=\frac{1}{4} I_{0} \lambda_{\mathrm{g}} \cdot(j \hat{x}+\hat{y}) \quad \text { for } \beta>0,
$$

leading to RHCP radiation for $\left.\mathbf{E}_{\mathrm{Brd}}\right|_{q=0.5} ^{n=1}$; this is not found with round model-C.

At second Hion frequency $f_{\mathrm{H} 2}$ (where $\beta>0, n=2$ ) and second Nion frequency (where $\beta<0, n=2$ ), $\left.K^{+}\right|^{n=2}=0$ and $\left.K^{-}\right|^{n=2}=0$ are obtained from equations (5) and (6), respectively, and hence, the polarization vector of equation (20) yields zero,

$$
\left.\mathbf{A}_{\text {st }}\right|_{q=0.5} ^{n=2,3}=0, \quad \text { for } \beta \gtrless 0,
$$


<smiles>[Y]C([Y])C([Y])[X]</smiles>

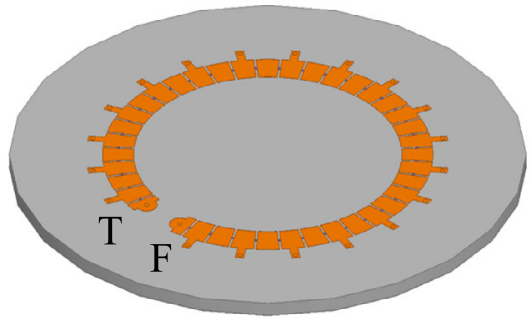

Fig. 14. Round metaloop-N antenna (RND-MTLP-N), made by curving the N-type metaline shown in Figure $2 \mathrm{~b}$, where $N_{\text {cell }}=19$.

resulting in no broadside radiation. This also happens at the $n$th Hion frequency, $f_{\mathrm{Hn}}$, and the $n$th Nion frequency, $f_{\mathrm{Nn}}$, for integers $n \geq 4$.

Based on the above-mentioned results, broadside radiation $\left.\mathbf{E}_{\mathrm{Brd}}\right|_{q=0.5} ^{n}$ for round model- $\mathrm{N}$ is depicted in Figure 13c. It is worth emphasizing that round model-N forms an LHCP wave at $f_{\mathrm{N} 1}$ and an RHCP wave at $f_{\mathrm{H} 1}$ when $q$ is not zero. However, this RHCP wave at $f_{\mathrm{H} 1}$ disappears when $q=1$, although the LHCP wave at $f_{\mathrm{N} 1}$ is present, as shown in Figure 13b.

\subsection{Round metaloop-N antenna (RND-MTLP-N): approximation of round model- $\mathrm{N}$}

Round model-N in Section 3.3 is approximated by curving the N-type metaline in Figure 2b, as shown in Figure 14. This antenna is referred to as the round metaloop-N antenna (RND-MTLP-N). The radiation occurs roughly between $f_{\mathrm{L}}$ and $f_{\mathrm{U}}$ shown in Figure 9 , because the N-type metaline is designed such that its dispersion characteristics are as similar as possible to those of the C-type metaline.

Figures $15 \mathrm{a}$ and $15 \mathrm{~b}$ show the frequency response of the gain and the VSWR, respectively. It is observed that the RND-MTLP-N has a small gain at a frequency near $f_{\mathrm{H} 1}$. This means that the RND-MTLP-N no longer has a deviation factor of $q=1$ (discussed in Sect. 3.3), due to the effects of the curved structure. The VSWR across $G_{\mathrm{L}}$ and $G_{\mathrm{R}}$ gain bandwidths for a 3 -dB gain-drop criterion is reasonably small, leading to good $50-\Omega$ impedance matching. Figures 16a and 16b show the radiation patterns when the gain is maximal at frequencies near $f_{\mathrm{N} 1}$ and $f_{\mathrm{H} 1}$, respectively. The radiated waves below and above transition frequency $f_{\mathrm{T}}=3 \mathrm{GHz}$ are LHCP and RHCP, respectively; this behaviour is similar to that observed in Figure 13c.

\section{Square metaloop antenna}

\subsection{Square model-C}

Figure 17 shows an antenna model made of a bent narrow width line, referred to as square model- $C$, where a traveling current, $\boldsymbol{I}\left(s^{\prime}\right)=I_{0} e^{-j \beta s^{\prime}} \hat{s}$, flows from point $F$ along the loop, with the same assumptions as those for the current in round model-C: unidirectional current with a constant amplitude,

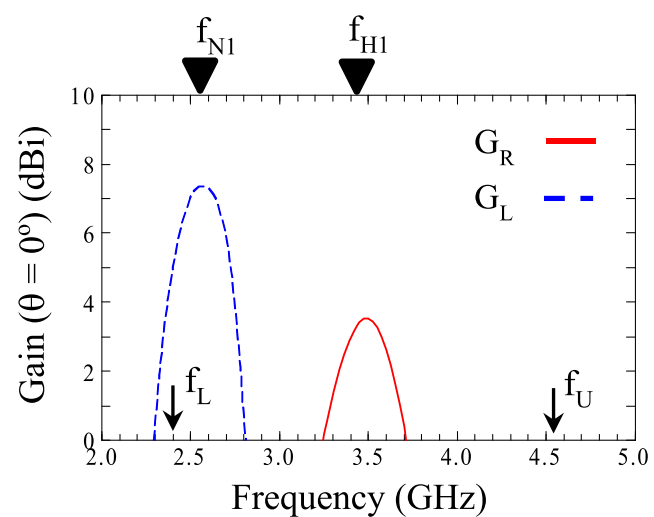

(a)

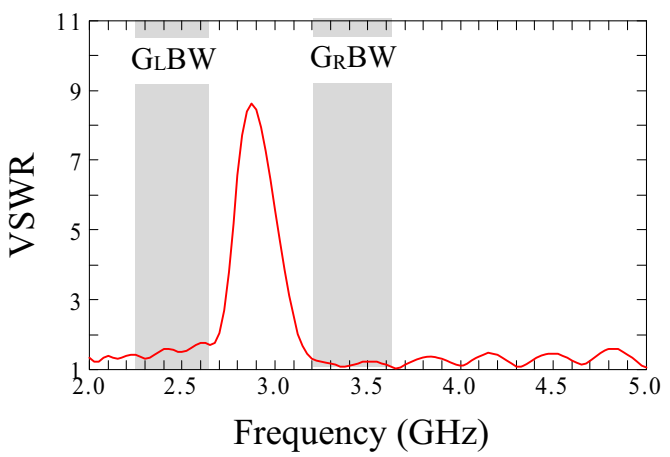

(b)

Fig. 15. Frequency response for the round metaloop-N antenna. $f_{\mathrm{N} 1}=2.55 \mathrm{GHz}$ and $f_{\mathrm{H} 1}=3.425 \mathrm{GHz}$. (a) Gain. (b) VSWR.

$I_{0}$, and phase constant $\beta$ that takes on either a positive or negative value.

The unit vector along the loop, $\hat{s}$, is for loop section 1 , $\hat{s}=-\hat{x}$ for loop section $2, \hat{s}=-\hat{y}$ for loop section $3, \hat{s}=\hat{x}$ for loop section 4 and $\hat{s}=\hat{y}$ for loop section 5 . Broadside radiation $\left.\mathbf{E}_{\mathrm{BrdSQ}}\right|^{n}$ formed by the $\hat{s}$-directed current along the loop is formulated to be

$$
\left.\mathbf{E}_{\mathrm{BrdSQ}}\right|^{n}=-\left.\frac{j \omega \mu}{4 \pi} \frac{e^{-j k R}}{R} \mathbf{B}_{\mathrm{s}}\right|^{n},
$$

where $\left.\mathbf{B}_{\mathrm{s}}\right|^{n}$ is referred to as the polarization vector for the $\hat{s}$-directed current:

$$
\begin{aligned}
\left.\mathbf{B}_{\mathrm{S}}\right|^{n}= & I_{0} \cdot \frac{\lambda_{\mathrm{g}}}{\pi} e^{\mp j n \pi} \cdot\left[\sin n \pi \hat{y}-2 \sin \frac{n \pi}{2} \cos \frac{n \pi}{4}\right. \\
& \left.\times\left(\hat{y} \pm j \tan \frac{n \pi}{4} \hat{x}\right)\right] \quad \text { for } \beta \gtrless 0
\end{aligned}
$$

with $n$ being a real number. Equation (25) becomes

$$
\begin{array}{ll}
\left.\mathbf{B}_{\mathrm{s}}\right|^{n=1}=\sqrt{2} I_{0} \cdot \frac{\lambda_{\mathrm{g}}}{\pi}[\hat{y} \pm j \hat{x}], & \text { for } \beta \gtrless 0 \\
\left.\mathbf{B}_{\mathbf{s}}\right|^{n=2}=0, & \text { for } \beta \gtrless 0 \\
\left.\mathbf{B}_{\mathrm{s}}\right|^{n=3}=\sqrt{2} I_{0} \cdot \frac{\lambda_{\mathrm{g}}}{\pi} \cdot[\hat{y} \mp j \hat{x}] & \text { for } \beta \gtrless 0 .
\end{array}
$$




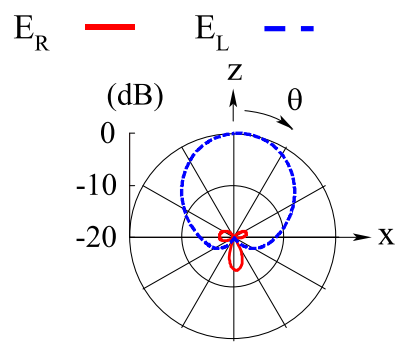

(a)

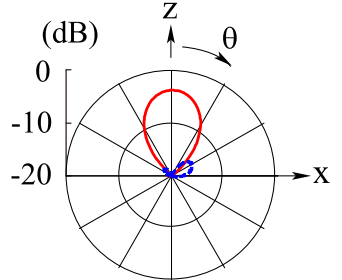

(b)
Fig. 16. Normalized radiation pattern for the round metaloop-N antenna. (a) At $2.575 \mathrm{GHz}$ near $f_{\mathrm{N} 1}=2.55 \mathrm{GHz}$. (b) At $3.5 \mathrm{GHz}$ near $f_{\mathrm{H} 1}=3.425 \mathrm{GHz}$.

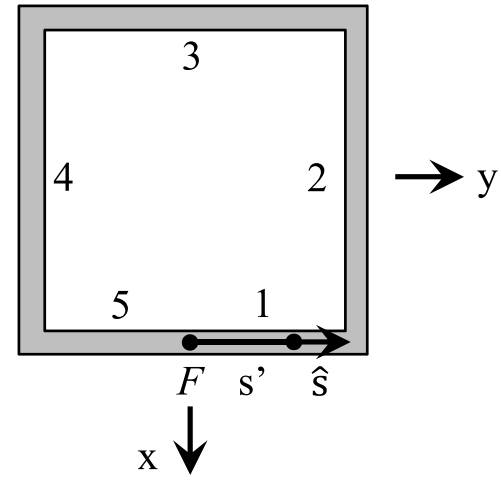

Fig. 17. Square model-C.

Equation (26) means that broadside radiation $\left.\mathbf{E}_{\mathrm{BrdSQ}}\right|^{n=1}$ is LHCP at first Nion frequency $f_{\mathrm{N} 1}$ and RHCP at first Hion frequency $f_{\mathrm{H} 1}$, while equation (27) reveals no broadside radiation at second Nion frequency $f_{\mathrm{N} 2}$ and second Hion frequency $f_{\mathrm{H} 2}$. As seen from equation (28), the broadside radiation is RHCP at third Nion frequency $f_{\mathrm{N} 3}$ and LHCP at third Hion frequency $f_{\mathrm{H} 3}$. The broadside radiation, $\left.\mathbf{E}_{\mathrm{BrdSQ}}\right|^{n}$, is illustrated in Figure 18. It should be emphasized that the generation of $\mathrm{CP}$ broadside radiation for square model-C differs from that of its counterpart, round model-C, at frequencies below $f_{\mathrm{N} 1}$ and above $f_{\mathrm{H} 1}$.

\subsection{Square metaloop-C antenna (SQR-MTLP-C): approximation of square model-C}

Square model-C is approximately realized by making square bends in the C-type metaline in Figure 2a. The antenna shown in Figure 19 is referred to as the square metaloop-C antenna or SQR-MTLP-C, whose parameters are given in Table 1.

Figure 20a and 20b show the frequency response of the gain and VSWR, respectively. The gain is maximal at frequencies near $f_{\mathrm{N} 1}$ and $f_{\mathrm{H} 1}$, both within the fast wave frequency region (from $f_{\mathrm{L}}$ to $f_{\mathrm{U}}$, where the radiation occurs), as expected from square model-C. Figure $20 \mathrm{~b}$ reveals that the VSWR within the $G_{\mathrm{L}}$ and $G_{\mathrm{R}}$ bandwidths for a 3 -dB gain-drop criterion is less than 2 , as with the RNDMTLP-C. Thus, the SQR-MTLP-C acts as a dual-band counter $\mathrm{CP}$ radiation element within the fast wave frequency

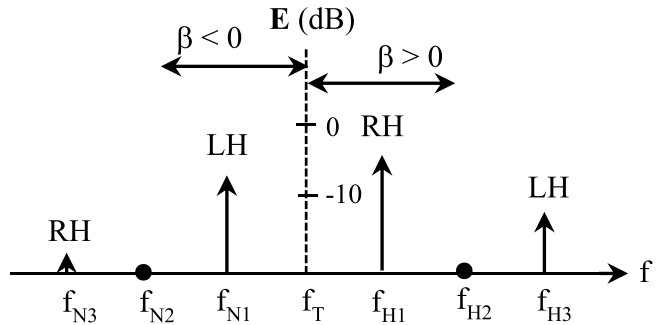

Fig. 18. Broadside radiation $\left.\mathbf{E}_{\mathrm{BrdSQ}}\right|^{n}=\mathbf{E}$ for square model-C.

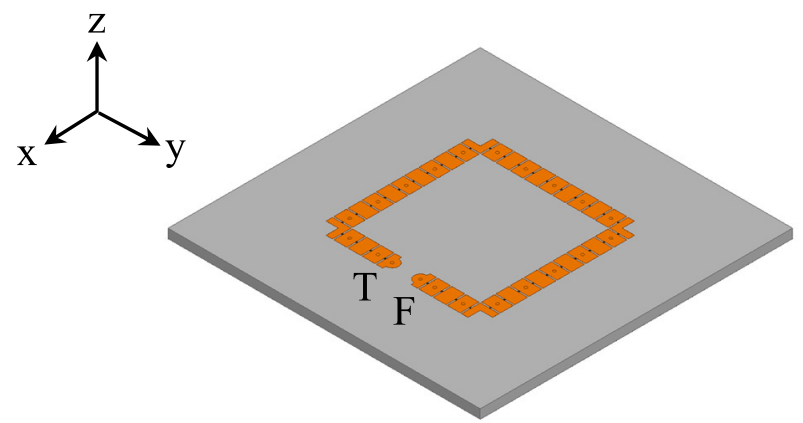

Fig. 19. Square metaloop-C antenna (SQR-MTLP-C), where $N_{\text {cell }}=19$.

region, as with the RND-MTLP-C, although square model$\mathrm{C}$ and round model-C behave differently at frequencies below $f_{\mathrm{N} 1}$ and above $f_{\mathrm{H} 1}$, as shown in Figures 18 and 7 .

The radiation patterns when the gain becomes maximal at frequency $f_{\text {GLmax }}$ near first Nion frequency $f_{\mathrm{N} 1}$ and frequency $f_{\mathrm{GR} \text { max }}$ near first Hion frequency $f_{\mathrm{H} 1}$ are shown in Figures $21 \mathrm{a}$ and $21 \mathrm{~b}$, respectively. The half-power beam width (HPBW) for the LHCP radiation is wider than that for the RHCP radiation. This difference leads to the same characteristic for the gain described in Section 3.1, i.e. the LHCP gain is smaller than the RHCP gain, as shown in Figure 20a.

\subsection{Square model-N}

The radiation model made of a bent wide width line, shown in Figure 22, is referred to as square model- $N$, corresponding to round model-N in Figure 12. The current on the loop is expressed as $\boldsymbol{I}\left(s^{\prime}\right)=I_{0}(\hat{\mathbf{s}}-j q \hat{\mathbf{t}}) e^{-j \beta s^{\prime}}$ with deviation factor $q$. Vector $\hat{\mathbf{t}}$ is the unit vector perpendicular to $\hat{\mathbf{s}}$, i.e. $\hat{\mathbf{t}}=\hat{x}$ for loop section $1, \hat{\mathbf{t}}=\hat{y}$ for loop section 2 , $\hat{\mathbf{t}}=-\hat{x}$ for loop section $3, \hat{\mathbf{t}}=-\hat{y}$ for loop section 4 and $\hat{\mathbf{t}}=\hat{x}$ for loop section 5 . The assumptions for the current are the same as those for round model-C in Section 3.1.

The radiation in the broadside direction, $\left.\mathbf{E}_{\mathrm{BrdSQ}}\right|_{q} ^{n}$, is formulated to be

$$
\left.\mathbf{E}_{\mathrm{BrdSQ}}\right|_{q} ^{n}=-\left.\frac{j \omega \mu}{4 \pi} \frac{e^{-j k R}}{R} \mathbf{B}_{\mathrm{st}}\right|_{q} ^{n},
$$

where the polarization vector due to $\hat{\mathbf{s}}$ - and $\hat{\mathbf{t}}$-directed currents is

$$
\left.\left.\mathbf{B}_{\mathbf{s t}}\right|_{q} ^{n} \equiv \mathbf{B}_{\mathbf{s t}}^{+}\right|_{q} ^{n}+\left.\mathbf{B}_{\mathbf{s t}}^{-}\right|_{q} ^{n}
$$




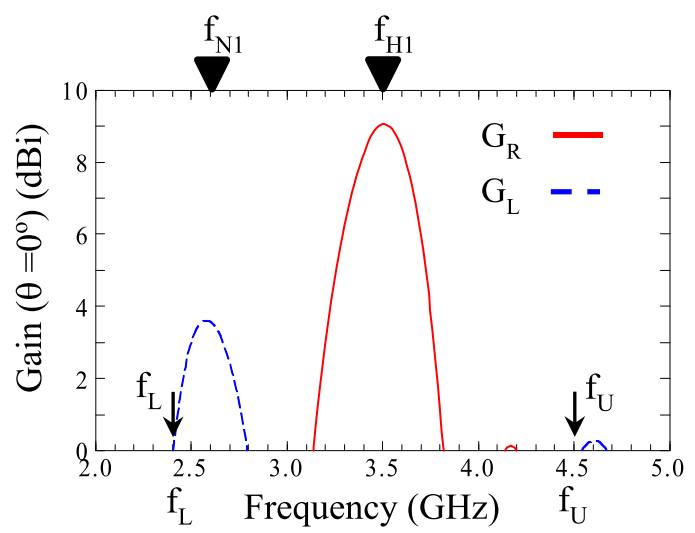

(a)

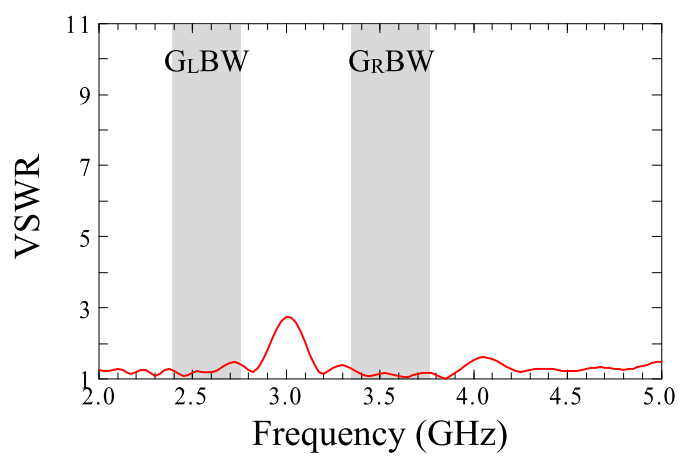

(b)

Fig. 20. Frequency response for the square metaloop-C antenna. (a) Gain. (b) VSWR.

with

$$
\begin{aligned}
\left.\mathbf{B}_{\mathrm{st}}^{+}\right|_{q} ^{n}= & \frac{1-q}{2} I_{0} \cdot \frac{\lambda_{\mathrm{g}}}{\pi} e^{\mp j n \pi}(j \hat{x}+\hat{y}) \\
& \times\left(\sin n \pi-2 \sin \frac{n \pi}{2} \cos \frac{n \pi}{4}\right) \cdot\left(1 \pm \tan \frac{n \pi}{4}\right)
\end{aligned}
$$$$
\text { for } \beta \gtrless 0
$$

$$
\begin{aligned}
\left.\mathbf{B}_{\mathrm{st}}^{-}\right|_{q} ^{n}= & \frac{1+q}{2} I_{0} \cdot \frac{\lambda_{g}}{\pi} e^{\mp j n \pi}(-j \hat{x}+\hat{y}) \\
& \times\left(\sin n \pi-2 \sin \frac{n \pi}{2} \cos \frac{n \pi}{4}\right) \cdot\left(1 \mp \tan \frac{n \pi}{4}\right)
\end{aligned}
$$

for $\beta \gtrless 0$.

When $q=0$, the current is $I\left(s^{\prime}\right)=I_{0} \hat{\mathbf{s}} \mathrm{e}^{-j \beta s^{\prime}}$ and equation (30) yields equation (25): $\left.\mathbf{B}_{\text {st }}\right|_{q=0} ^{n}=\left.\mathbf{B}_{s}\right|^{n}$. Hence, equations (26)-(28) are obtained, resulting in broadside radiation $\left.\mathbf{E}_{\text {BrdSQ }}\right|_{q=0} ^{n}$, shown in Figure 23a.

Next, we consider $\left.\mathbf{E}_{\mathrm{BrdSQ}}\right|_{q} ^{n}$ when $q=1$ in equation (29). Equation (31) becomes zero: $\left.\mathbf{B}_{\text {st }}^{+}\right|_{q=1} ^{n}=0$. Hence, equation (29) is written as

$$
\left.\mathbf{E}_{\mathrm{BrdSQ}}\right|_{q=1} ^{n}=-\left.\frac{j \omega \mu}{4 \pi} \frac{e^{-j k R}}{R} \mathbf{B}_{\mathrm{st}}^{-}\right|_{q=1} ^{n}
$$

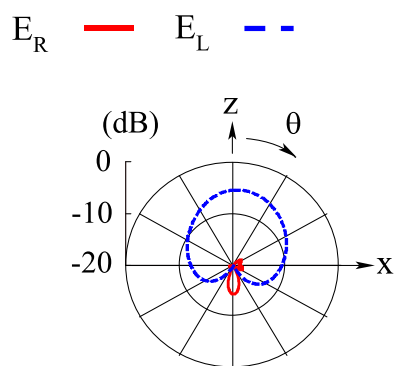

(a)

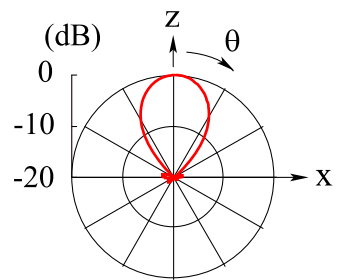

(b)
Fig. 21. Normalized radiation pattern for the square metaloop-C antenna. (a) At frequency $f_{\text {GLmax }}=2.58 \mathrm{GHz}$ near $f_{\mathrm{N} 1}=2.60 \mathrm{GHz}$. (b) At frequency $f_{\mathrm{GRmax}}=3.51 \mathrm{GHz}$ near $f_{\mathrm{H} 1}=3.50 \mathrm{GHz}$.

where $\left.\mathbf{B}_{\mathbf{s t}}^{-}\right|_{q=1} ^{n}$ for $n=1,2$, and 3 is

$$
\begin{array}{ll}
\left.\mathbf{B}_{\text {st }}^{-}\right|_{q=1} ^{n=1}=\sqrt{2} I_{0} \cdot \frac{\lambda_{\mathrm{g}}}{\pi}(-j \hat{x}+\hat{y})(1 \mp 1) & \text { for } \beta \gtrless 0 \\
\left.\mathbf{B}_{\text {st }}^{-}\right|_{q=1} ^{n=2}=0, & \text { for } \beta \gtrless 0 \\
\left.\mathbf{B}_{\text {st }}^{-}\right|_{q=1} ^{n=3}=\sqrt{2} I_{0} \cdot \frac{\lambda_{\mathrm{g}}}{\pi}(-j \hat{x}+\hat{y})(1 \pm 1), & \text { for } \beta \gtrless 0 .
\end{array}
$$

The broadside radiation for these results, $\left.\mathbf{E}_{\mathrm{BrdSQ}}\right|_{q=1} ^{n}$, is shown as Figure 23b.

As performed for round model-N, an arbitrary case where deviation factor $q$ is neither 0 nor 1 is considered, choosing $q=0.5$ as one example. When $n=1$, equations (31) and (32) become

$$
\begin{array}{ll}
\left.\mathbf{B}_{\text {st }}^{+}\right|_{q=0.5} ^{n=1}=\frac{\sqrt{2}}{4} I_{0} \cdot \frac{\lambda_{\mathrm{g}}}{\pi}(j \hat{x}+\hat{y})(1 \pm 1), \quad \text { for } \beta \gtrless 0, \\
\left.\mathbf{B}_{\text {st }}^{-}\right|_{q=0,5} ^{n=1}=\frac{3 \sqrt{2}}{4} I_{0} \cdot \frac{\lambda_{\mathrm{g}}}{\pi}(-j \hat{x}+\hat{y})(1 \mp 1), \quad \text { for } \beta \gtrless 0 .
\end{array}
$$

Hence, equation (30) for $n=1$ and $q=0.5$ becomes

$$
\begin{aligned}
& \left.\mathbf{B}_{\mathrm{st}}\right|_{\mathrm{q}=0.5} ^{n=1}=\frac{\sqrt{2}}{2} I_{0} \cdot \frac{\lambda_{\mathrm{g}}}{\pi}(j \hat{x}+\hat{y}), \quad \text { for } \beta>0 \\
& \left.\mathbf{B}_{\mathrm{st}}\right|_{q=0.5} ^{n=1}=\frac{3 \sqrt{2}}{2} I_{0} \cdot \frac{\lambda_{\mathrm{g}}}{\pi}(-j \hat{x}+\hat{y}), \quad \text { for } \beta<0 .
\end{aligned}
$$

When $n=2$,

$$
\begin{aligned}
& \left.\mathbf{B}_{\text {st }}^{+}\right|_{q=0.5} ^{n=2}=0, \quad \text { for } \beta \gtrless 0 \\
& \left.\mathbf{B}_{\text {st }}^{-}\right|_{q=0.5} ^{n=2}=0, \quad \text { for } \beta \gtrless 0 .
\end{aligned}
$$

In addition, when $n=3$,

$$
\left.\mathbf{B}_{\text {st }}^{+}\right|_{q=0.5} ^{n=3}=\frac{\sqrt{2}}{4} I_{0} \cdot \frac{\lambda_{\mathrm{g}}}{\pi}(j \hat{x}+\hat{y})(1 \mp 1), \quad \text { for } \beta \gtrless 0
$$




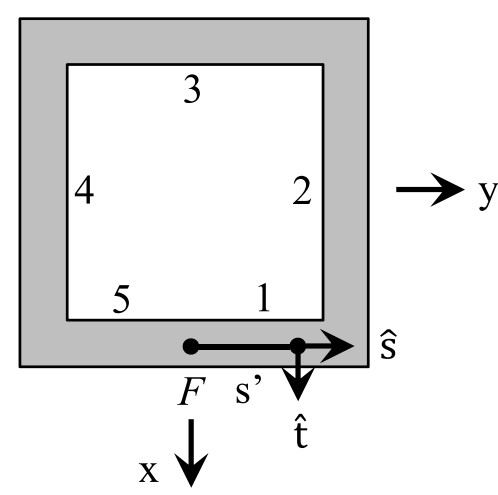

Fig. 22. Square model-N.

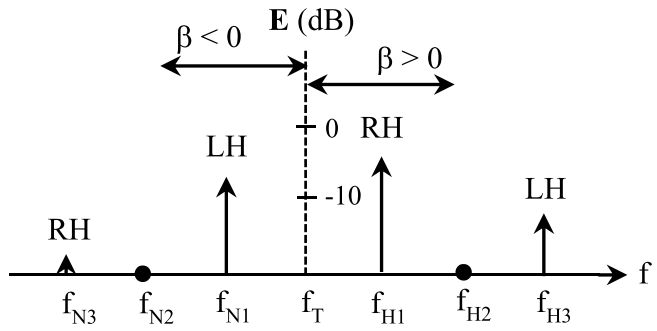

(a)

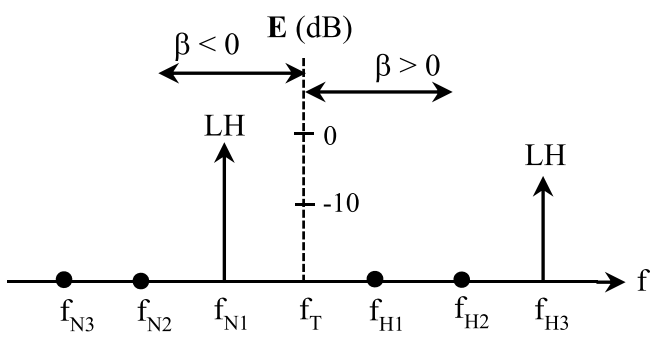

(b)

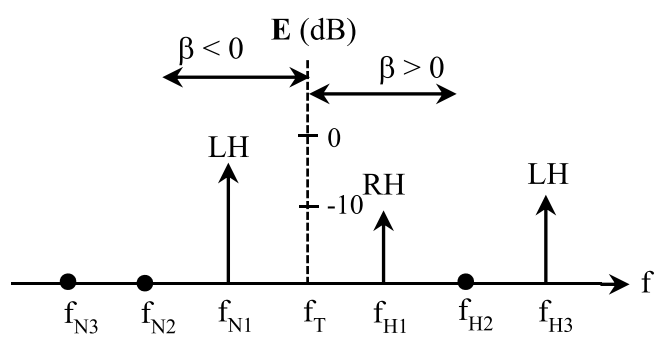

(c)

Fig. 23. $\left.\mathbf{E}_{\mathrm{BrdSQ}}\right|_{q} ^{n}=\mathbf{E}$ for square model-N. (a) $q=0$. (b) $q=1$. (c) $q=0.5$.

$\left.\mathbf{B}_{\mathrm{st}}^{-}\right|_{q=0.5} ^{n=3}=\frac{3 \sqrt{2}}{4} I_{0} \cdot \frac{\lambda_{\mathrm{g}}}{\pi}(-j \hat{x}+\hat{y})(1 \pm 1), \quad$ for $\beta \gtrless 0$.

The sum of equations (43) and (44) leads to

$$
\begin{aligned}
& \left.\mathbf{B}_{\mathbf{s t}}\right|_{q=0.5} ^{n=3}=\frac{3 \sqrt{2}}{2} I_{0} \cdot \frac{\lambda_{\mathrm{g}}}{\pi}(-j \hat{x}+\hat{y}), \quad \text { for } \beta>0 \\
& \left.\mathbf{B}_{\mathbf{s t}}\right|_{q=0.5} ^{n=3}=\frac{\sqrt{2}}{2} I_{0} \cdot \frac{\lambda_{\mathrm{g}}}{\pi}(j \hat{x}+\hat{y}), \quad \text { for } \beta<0 .
\end{aligned}
$$

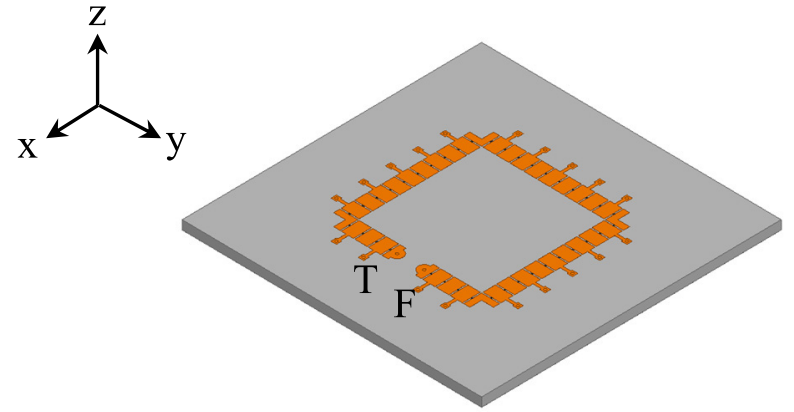

Fig. 24. Square metaloop-N antenna or SQR-MTLP-N, where $N_{\text {cell }}=19$.

Using the above results, broadside radiation $\left.\mathbf{E}_{\mathrm{BrdSQ}}\right|_{q=0.5} ^{n}$ is summarized in Figure 23c.

\subsection{Square metaloop-N antenna (SQR-MTLP-N): approximation of square model- $\mathrm{N}$}

Figure 24 shows an antenna that approximates square model-N discussed in Section 4.3. This antenna, referred to as the square metaloop- $N$ antenna or SQR-MTLP-N, is formed by bending the N-type metaline in Figure $2 \mathrm{~b}$. The formed metaloop is composed of five arm sections having strips that support the flow of $\hat{\mathbf{t}}$-directed current. The configuration parameters for the SQR-MTLP-N are summarized in Table 1.

It is difficult to realize $q=0$ and 1 for the square-bent metaline due to mutual coupling between the arm filaments, as with the RND-MTLP-N. Therefore, it is inferred from Figure $23 \mathrm{c}$ that, as the frequency is increased, the broadside radiation will change from LHCP to RHCP and then to LHCP. This is confirmed by the frequency response of the tri-band gain shown in Figure 25a. It should be emphasized that the tri-band gain characteristic obtained from the SQR-MTLP-N cannot be realized by its counterpart, the RND-MTLP-N (see Fig. 15a). Also, it should be emphasized that the VSWR within the three bands is desirably small, as shown in Figure 25b. The change in the principal component of the radiation pattern with increase in frequency (from $E_{\mathrm{L}}$, through $E_{\mathrm{R}}$, to $E_{\mathrm{L}}$ ), shown in Figure 26, is consistent with the change in the components of the tri-band gain (from $G_{\mathrm{L}}$, through $G_{\mathrm{R}}$, to $\left.G_{\mathrm{L}}\right)[13]$.

Note that the upper band-edge frequency for the fast wave, $f_{\mathrm{U}}$, and the third Hion frequency $f_{\mathrm{H} 3}$ in Figure $25 \mathrm{a}$ are the values (original values) obtained when the metaline is straight. The position of $f_{\mathrm{U}}$ and $f_{\mathrm{H} 3}$ is affected when the metaline is bent, due to mutual coupling; in other words, guided wavelength $\lambda_{\mathrm{g}}$ is changed. This adds an error component to the position of $f_{\mathrm{U}}$ and $f_{\mathrm{H} 3}$. A true upper bandedge frequency and a true third Hion frequency become larger than the original values, because the maximum LHCP gain appearing on the right side in Figure 25a is the gain due to the radiation at the true third Hion frequency $f_{\mathrm{H} 3}$. 


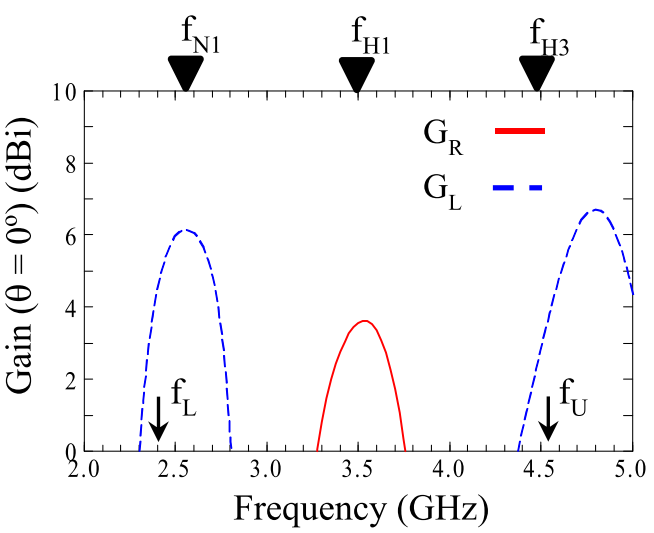

(a)

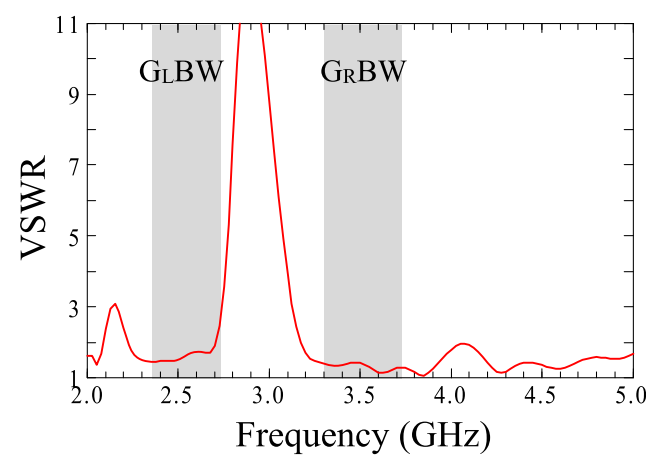

(b)

Fig. 25. Frequency response for the square metaloop- $\mathrm{N}$ antenna (SQR-MTLP-N). (a) Gain. (b) VSWR.

\section{Conclusions}

The linearly polarized C-type metaline and circularly polarized N-type metaline have been designed such that their dispersion diagrams are as similar as possible. The polarization for round model-C has been analysed and the RND-MTLP-C antenna, which is an approximation of model-C and implemented using a C-type metaline, has been investigated. As expected from the analysis results of round model-C, the RND-MTLP-C antenna acts as a dual-band counter $\mathrm{CP}$ radiation element across a fast wave frequency region. In addition, the polarization for round model-N has been analysed. Subsequently, the RND-MTLP-N antenna, which is an approximation of model-N and implemented using an N-type metaline, has been investigated across the fast wave frequency region. It is found that the RND-MTLP-N antenna has principal LHCP radiation at frequencies near $f_{\mathrm{N} 1}$ and additional low-level RHCP radiation at frequencies near $f_{\mathrm{H} 1}$.

Similar investigation has been performed for square metaloop antennas SQR-MTLP-C and SQR-MTLP-N after analysing square model-C and square model-N, respectively. Consistent with the model analysis, the SQR-MTLP-C acts as a dual-band counter CP radiation element across a fast wave frequency region. An interesting point is that the SQR-MTLP-N acts as a tri-band CP

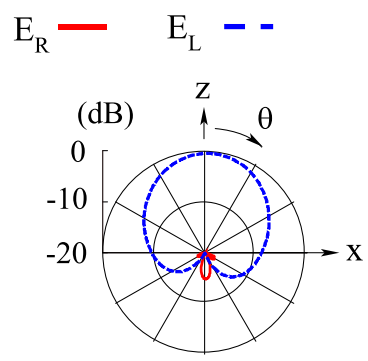

(a)

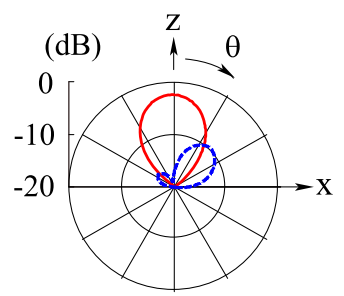

(b)

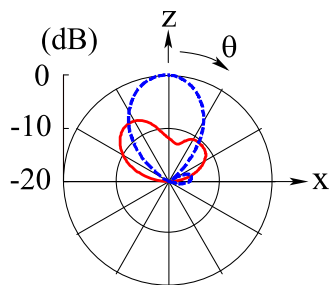

(c)

Fig. 26. Radiation pattern for the square metaloop- $\mathrm{N}$ antenna (SQR-MTLP-N). (a) At $2.55 \mathrm{GHz}=f_{\mathrm{N} 1}$. (b) At $3.55 \mathrm{GHz}$ near $f_{\mathrm{H} 1}=3.475 \mathrm{GHz}$. (c) At $4.8 \mathrm{GHz}$.

radiation element when deviation factor $q$ is neither zero nor one. Such behaviour cannot be found in the counterpart antenna, i.e. the RND-MTLP-N antenna.

\section{References}

1. L.W. Rispin, D.C. Chang, Wire and loop antennas, in: Y.T. Lo, S.W. Lee (Eds.), Antenna Handbook (Van Nostrand Reinhold Company Inc., New York, 1988)

2. H. Nakano, A numerical approach to line antennas printed on dielectric materials, Comput. Phys. Commun. 68, 441 (1991)

3. S.A. Rezaeieh, M.A. Antoniades, A.M. Abbosh, Gain enhancement of wideband metamaterial-loaded loop antenna with tightly coupled arc-shaped directors, IEEE Trans. Antennas Propag. 65, 2090 (2017)

4. X. Zhao, Y. Lee, K.Y. Jung, J.H. Choi, Design of a metamaterial-inspired size-reduced wideband loop antenna with frequency scanning characteristic, IET Microw. Antennas Propag. 6, 1227 (2012)

5. Y. Zhang, K. Wei, Z. Zhang, Y. Li, Z. Feng, A compact dual-mode metamaterial-based loop antenna for pattern diversity, IEEE Antennas Wirel. Propag. Lett. 14, 394 (2015)

6. S.A. Rezaeieh, M.A. Antoniades, A.M. Abbosh, Bandwidth and directivity enhancement of loop antenna by nonperiodic distribution of mu-negative metamaterial unit cells, IEEE Trans. Antennas Propag. 64, 3319 (2016)

7. C. Caloz, T. Itoh, Electromagnetic Metamaterials (John Wiley \& Sons, Inc., New York, 2006)

8. N. Engheta, R.W. Ziolkowski, Metamaterials (John Wiley \& Sons, Inc., New York, 2006) 
9. G.V. Eleftheriades, K.G. Balmain, Negative-Refraction Metamaterials: Fundamental Principles and Applications (John Wiley \& Sons, Inc., New York, 2005)

10. H. Nakano, J. Miyake, T. Sakurada, J. Yamauchi, Dual-band counter circularly polarized radiation from a single-arm metamaterial-based spiral antenna, IEEE Trans. Antennas Propag. 61, 2938 (2013)
11. H. Nakano, K. Yoshida, J. Yamauchi, Radiation characteristics metaloop antenna, IEEE Antennas Wirel. Propag. Lett. 12, 861 (2013)

12. E. Yamashita, Analysis Methods for Electromagnetic Wave Problems (Artech House, Boston, 1996)

13. H. Nakano, T. Yoshida, J. Yamauchi, Triband metaloop antenna, IEEE Antennas Wirel. Propag. Lett. 16, 1981 (2017)

Cite this article as: Hisamatsu Nakano, Tomoki Abe, Junji Yamauchi, Quasi-theoretical investigation of four circularly polarized metaloop antennas, EPJ Appl. Metamat. 6, 2, (2019) 